\title{
Closing the loop on modelling's role in natural resource investment planning
}

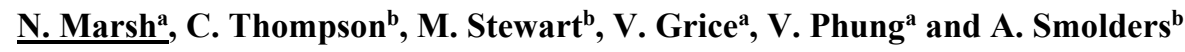 \\ ${ }^{a}$ Truii Pty Ltd, Brisbane Queensland, ${ }^{b}$ Seqwater, Ipswich, Queensland, \\ Email: nick.marsh@truii.com
}

\begin{abstract}
A perennial problem of natural resource management is how to most effectively use a limited budget to achieve the greatest environmental and social benefit. The field of water quality and environmental modelling has provided great insights to allow the consequences of alternative investment strategies to be investigated. However, the modelling process is largely divorced from the decision-making process. Policy makers and planners indirectly use the outputs from models in the planning process. The planned investment then becomes operationalized via on-ground investment plan. The end result is an on-ground implementation that is two steps removed from the modelling process and there is no ability to easily integrate the 'asconstructed' implementation as a modelled scenario. We propose to close the loop in the modelling-planningoperationalise workflow sequence. We illustrate the workflow approach using a recently developed web tool, based on simulated annealing and combining numerical and spatial data, used by Seqwater; the Catchment Investment Decision Support System.

Our approach in developing the Catchment Investment Decision Support System has been to create a single platform that completes tasks for different user groups across the natural resource management investment planning workflow (Figure 1). To ensure the application is fit for purpose for the users, we have restricted access to various components of the platform based on a user's roles. The platform has been designed for external and internal stakeholders, policy level staff, catchment modellers and operational staff who implement on-ground activities. The platform can be used to store data, create optimal intervention scenarios, create pragmatic versions of scenarios, publish web based visualisations of those scenarios for a broad audience, develop on-ground work plans and integrate 'as constructed' activities back into the model input data.
\end{abstract}

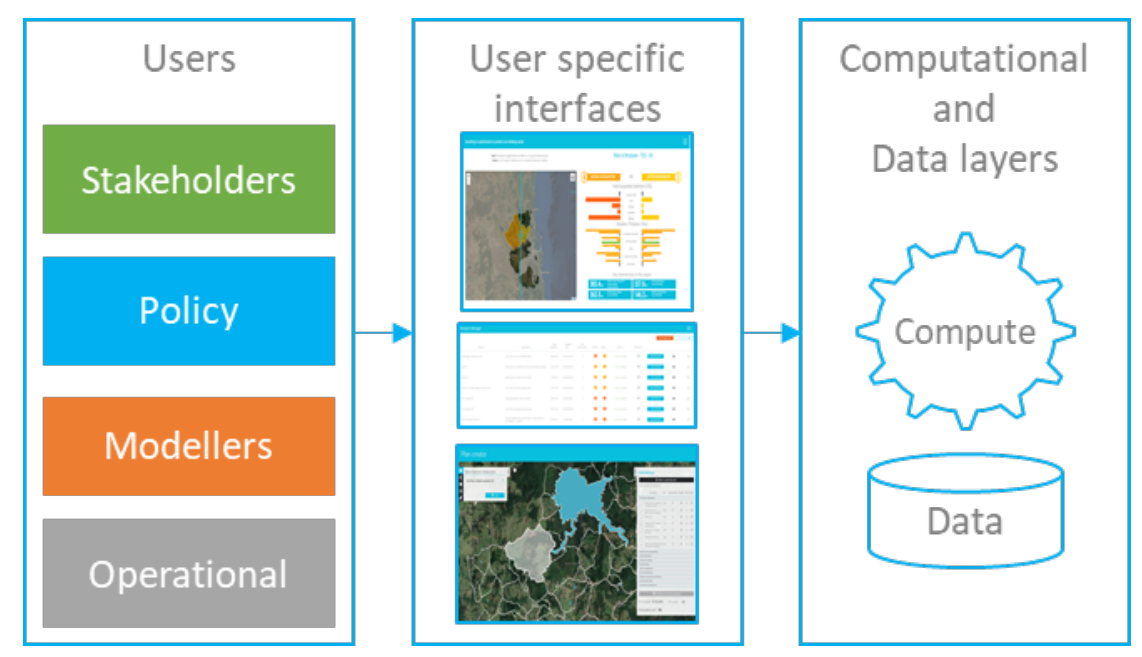

Figure 1. User roles based approach to access interfaces and computational and data layers of the platform

Keywords: Catchment investment, optimisation, workflow, operationalise, drinking water quality 


\section{INTRODUCTION}

The collective Australian investment in Natural Resource Management (NRM) activity across federal, state and private funding sources is of the order of \$3.5B/annum (RMCG 2017, ABS 2007). The financial investment is large, but it is smeared across a huge number of multifaceted environmental and social problems such that the resources available per project is very limited. How do the administrators of this investment assess the most worthwhile projects? A 2015 review of Australia's 2010-2030 Biodiversity Conservation Strategy found that the approach of the conservation strategy has failed to "effectively guide the efforts of governments, other organisations or individuals" (DOE 2016). Similar findings were reported from an audit of Queensland Government investment for managing water quality in Great Barrier Reef catchments (QAO 2015). There has not been an appropriate framework followed and therefore tools used to link policies to effective on-ground actions to help answer the question "what should I do and where should I do it?"

There are now several decision support frameworks such as strategic foresight, systematic conservation planning, structured decision making, open standards and evidence-based practice that can be used to guide operational planning (Schwartz et al., 2018) and selection of tools to address the questions of what actions are likely to achieve set objectives and where should the actions be applied? For example, SCaRPA DSS (Herron et al., 2008) combines systematic conservation planning and structured decision making frameworks for Catchment Management Authorities in New South Wales. SCaRPA DSS consists of multiple tools and/or models for identifying catchment scale priority areas for investment and ranking property scale project proposals against environmental targets for allocation of funding (Summerell et al., 2011).

These recently developed decision support tools support the NRM investment decision making process by including a quantitative modelling step in the decision-making process. We propose a more explicit and direct connection to a modelling approach through the use of a platform-based approach which is model centric. The platform should allow direct interaction with models or their outputs to inform decision making, and ultimately for on-ground implementation to feedback into the model boundary conditions.

Where modelling has been used to support the NRM investment process, the model output is typically an input into the planning process.

The basic (very simplified) workflow when using models to support NRM investment planning, includes modelling as a step in a process that is conducted independently of the policy maker. The process is mostly linear with limited feedback steps (Figure 2). We suggest an alternative approach, whereby the policy maker is in control of the modelling process. Furthermore, we suggest a series of feedback loops and refinements to capture the entire NRM investment planning

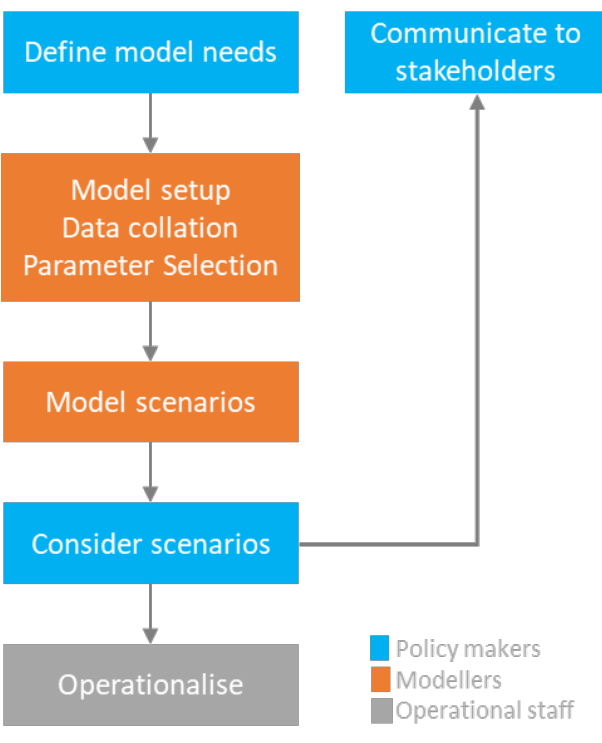

Figure 2. Basic workflow of NRM investment planning workflow (Figure 3).

The following sections step through the key steps in 3 of the modelling process using the Catchment Investment Decision Support System (CIDSS) as a case study to illustrate how the steps may be implemented.

Catchment Investment Decision Support System (CIDSS)

The Seqwater CIDSS has been developed to support the planning and implementation processes for investing in the mitigation of catchment derived risks to the quality of raw water received at water treatment plants from 40+ catchments in south east Queensland. The CIDSS considers 50+ potential onground interventions and uses simulated annealing to optimise their implementation based on most efficient reduction in risk to drinking water quality from sediment and microbial pollution. The CIDSS allows the post processing refinement of optimal scenarios as well as the development of implementation plans. 


\section{OPTIMISATION VS PRAGMATISATION}

In the development of the CIDSS we first consider the basic approaches of NRM investment tools. These tools used to support the planning of NRM investment typically either;

1) Optimise: Generate an optimal investment strategy based on a series of boundary conditions and a well-defined cost function, or

2) Pragamatise: Predict an environmental response given a fixed scenario representing a specific intervention strategy,

For the case of developing optimal intervention configurations, the model outputs lack realism because of the necessary simplifications of

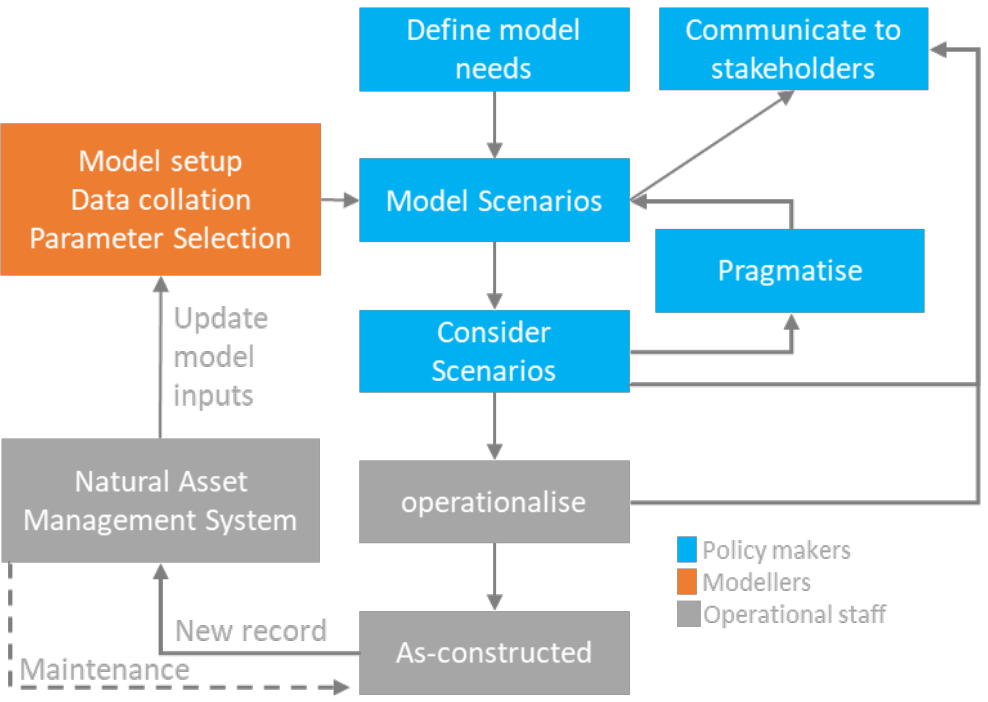

Figure 3. Proposed approach including feedback loops and direct modelling by policy makers

the optimisation process. For example, the optimisation process cannot easily incorporate social factors such as opportunities for co-investment or poorly numerated co-benefits. It would be useful for an optimal distribution of interventions to be post processed and adjusted to test the impact of social factors and perceived co-investment opportunities.

For the case of pragmatic scenario generation, the scenario creation and model run process is typically restricted to a few scenarios, with limited opportunity for iteration and refinement. This restricted number of scenarios is largely due to the separation of business units between modellers and policy maker and the subsequent high time costs of communication the appropriate configuring of new scenarios between business units. It would be useful to reduce the time cost of scenario generation by providing a more direct path (such as a scenario generation interface in a modelling tool) for policy makers to directly define alternative scenario configurations.

The approach to scenario development and refinement adopted in the CIDSS is to embrace both an optimisation approach and a pragmatic expert-driven scenario generation approach. Firstly an optimal solution is developed (simulated annealing approach). We consider this optimal solution as the 'base scenario' which is then refined interactively by planners by increasing or decreasing the various on-ground interventions in line with realistic opportunities. We call this step 'pragmatisation' and is represented as a return loop in Figure 3.

\section{DEFINING MODEL NEEDS}

An early step in defining the NRM investment planning process defining the modelling needs (top box in Figure 3). These modelling needs will consider the range of potential on-ground interventions to consider, the budget and the measures of success (such as water quality parameters and targets). The policy maker typically has the role of defining the boundary conditions of the NRM investment approach. This is analogous to a business analyst role. This business analyst role requires the collation and understanding of business interests and communicating the requirements to define the data requirements and inform the selection of a fit-forpurpose modelling paradigm.

The CIDSS adopts a fixed modelling approach of a spatially explicit non-timestepping model which is appropriate for creating a relative rank of investment strategies but not for accurate load prediction. The CIDSS can be set up for different measures, although the base consideration used by Seqwater is for suspended sediment and microbial contaminants. Importantly, the CIDSS is designed to determine which intervention will have the greatest influence at a strategic location downstream rather than focusing only on a hazard point source or potential load into a waterway. That is, given the purpose of Seqwater investing in on-ground works in catchments is to help ensure the safety of drinking water at the point of supply, the relative rank of an investment strategy is based on its impact on the quality of raw water received at water treatment plants each with differing treatment capabilities. 


\section{COMMUNICATING TO STAKEHOLDERS}

A further role of the policy maker is to communicate back to stakeholders on the NRM investment process. The approach adopted by the CIDSS for the communication process is to allow the policy maker to select a 'featured scenario' for display on a generally accessible interface to allow other members of the organization to interactively explore the scenario results (Figure 4). This approach allows the end audience who are affected by the NRM decision making process to directly interact with the model results and familiarize themselves with the opportunities and limitations of the modelling approach.

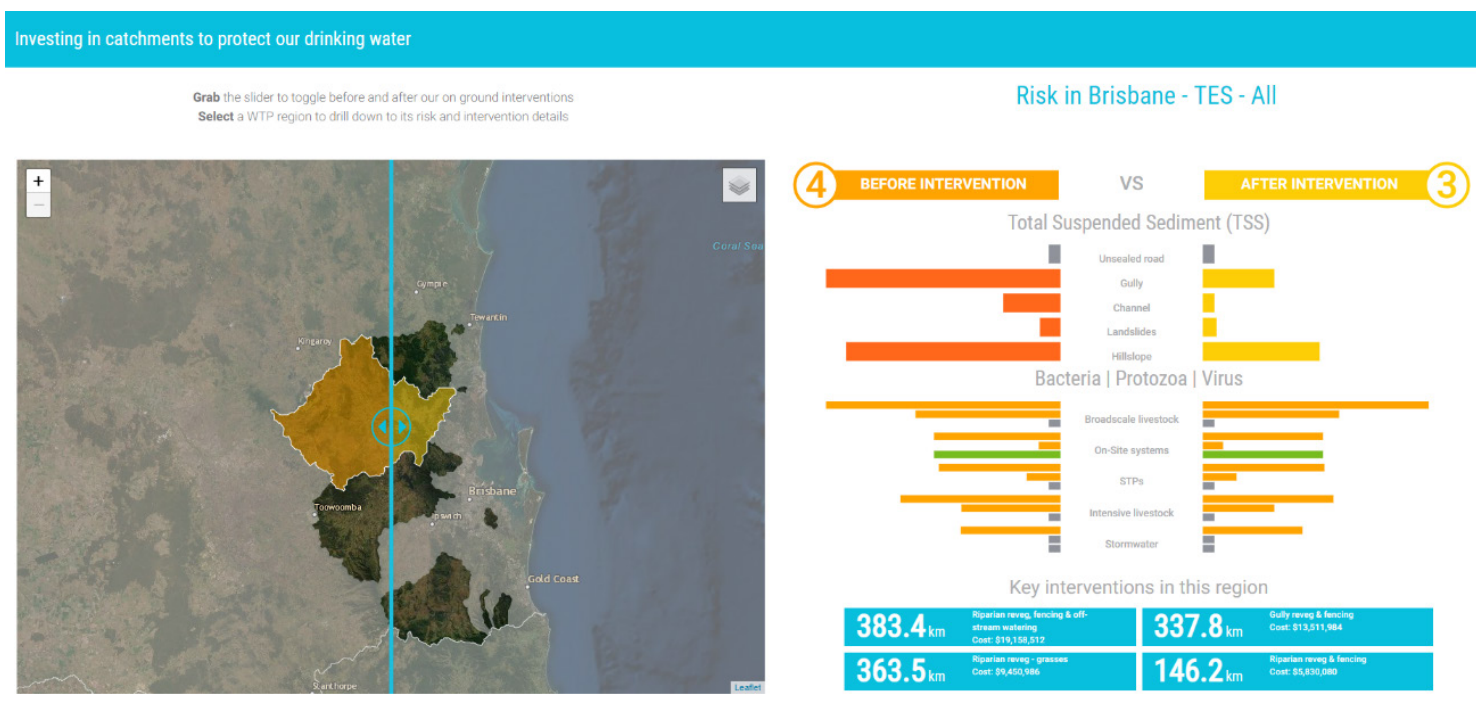

Figure 4. Featured scenario interface to communicate the model scenario to stakeholders

\section{MODEL SCENARIOS}

The 'model scenarios' step in Figures 2 and 3 requires the collation and parameterisation of a model environment. Traditionally the running of scenarios is conducted entirely by a modeler. The CIDSS approach is for the modeler to provide the base parameterisation and calibration and to provide a range of scenario boundary conditions. The CIDSS includes an interface to allow policy makers to create new scenarios within the bounds defined by the modeler.

More likely, policy makers may wish to compare scenarios and familiarize themselves with the underlying scenario configuration. To this end the CIDSS has a scenario management library to allow access to the underlying settings for each scenario.

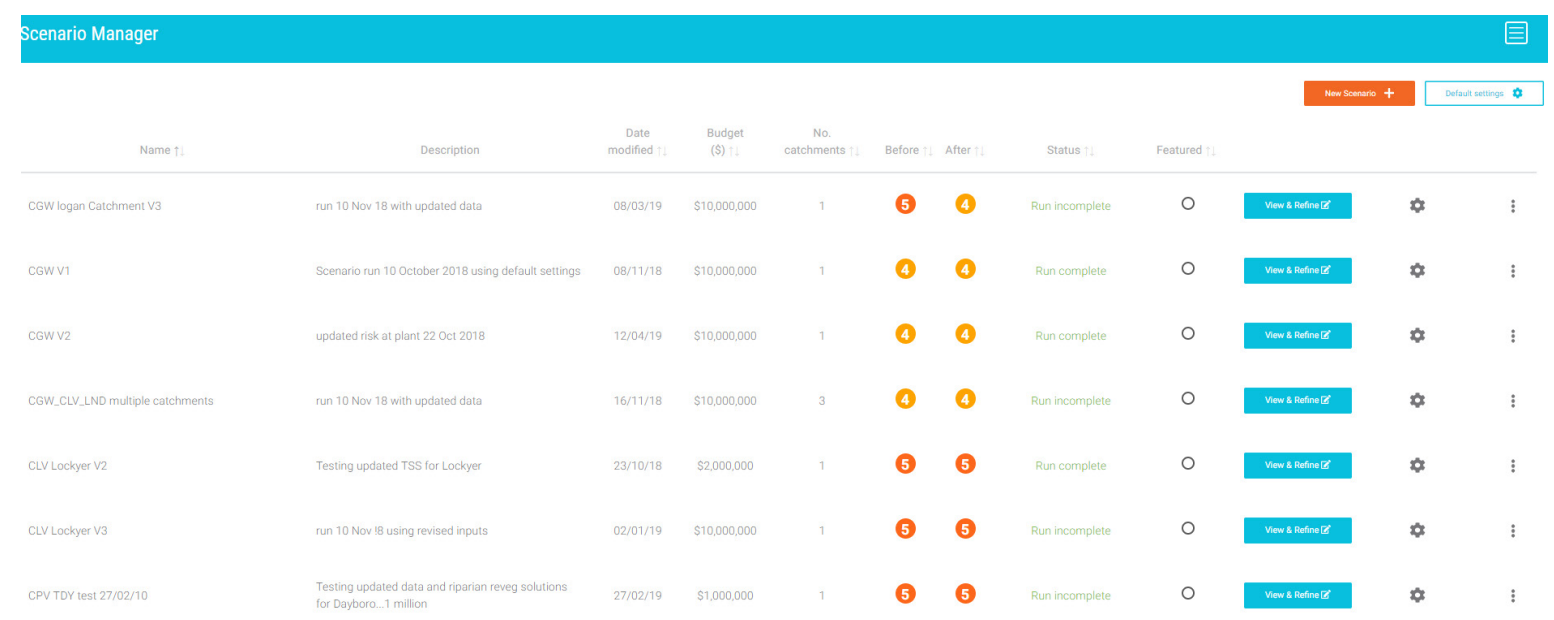

Figure 5. Scenario management library to allow policy makers to explore scenario settings and compare scenario results. 


\section{PRAGMATISATION}

The CIDSS software produces an optimal distribution of interventions for a given budget. These theoretical optimum results are rarely in a form that represents an implementable solution. Optimal solutions are at best an academic exercise. The optimal solution needs to be refined to reflect broader opportunities and co-benefits not otherwise captured in the modeling process. Rather than further complicate the optimization process by trying to capture these inconsistently defined constraints, the CIDSS approach has been to provide a postprocessing step of 'pragmatising' the result. This pragmatisation step adopts the optimal solution as a base case for the definition of a scenario. The policy maker can add and remove interventions at different spatial scales to reflect broader opportunities.
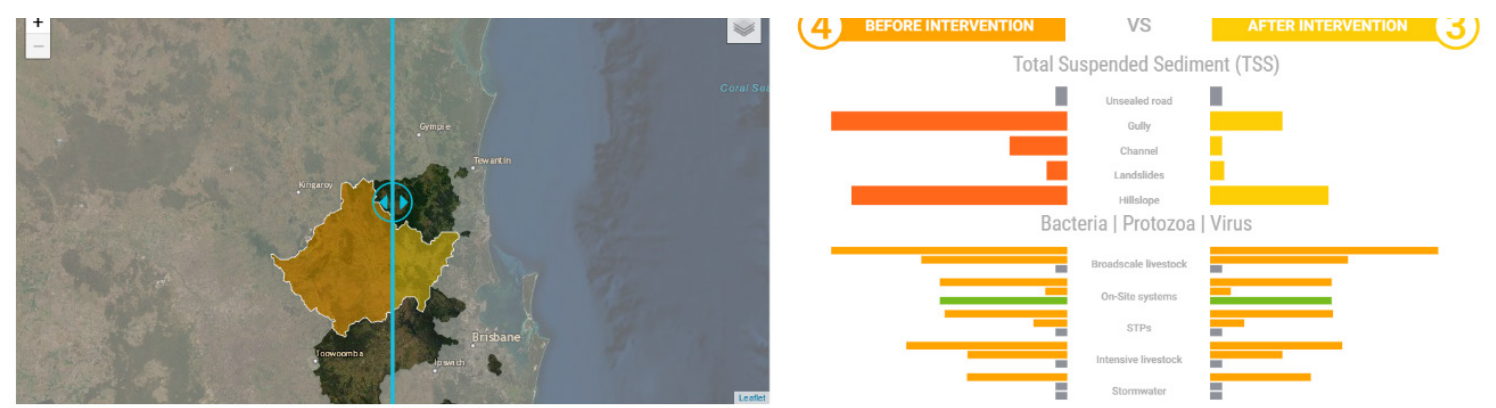

Budget: $\$ 80,000,000$ | Scenario calculated cost: $\$ 79,971,566$

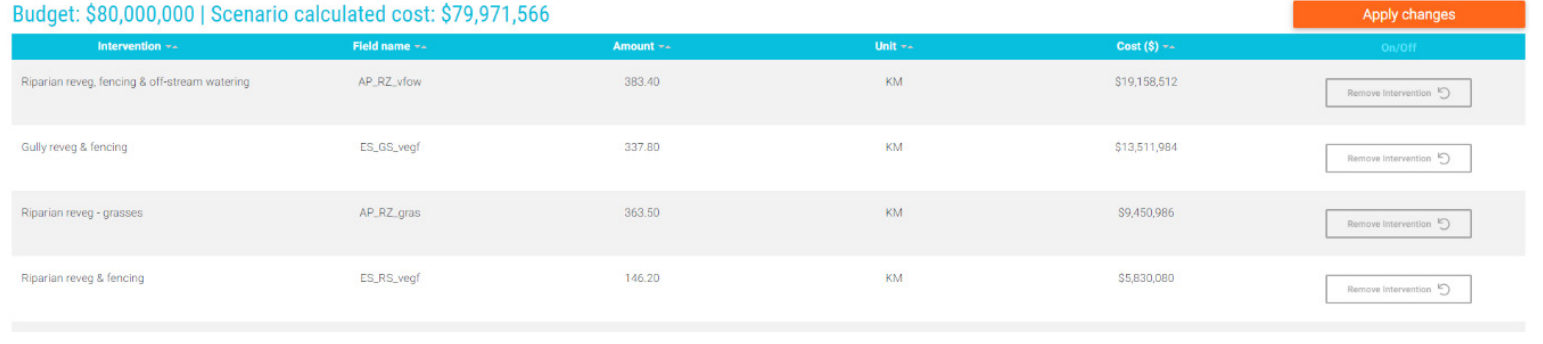

Figure 6. The pragmatisation interface for CIDSS (termed view and refine)

\section{OPERATIONALISATION}

After a series of pragmatisation iterations involving different stakeholders, the policy maker arrives at a scenario that illustrates a fair representation of a given investment strategy (e.g. 5 year plan / annual plan) to reduce the risk to water quality received at a particular water treatment plant to an acceptable level. The next step in the process is to convert this plan into specific projects that can be operationalized with on-ground implementation plans. The CIDSS operates at a planning unit spatial scale, for Seqwater these planning units have been developed through hydro geomorphological assessment and are typically $1-50 \mathrm{~km}^{2}$ in size. An operational project plan will need to prescribe where interventions can occur down to the property scale. The 
approach adopted by CIDSS is to provide an annotated map interface to allow the creation of operational plans (overlayed on a selected scenario).

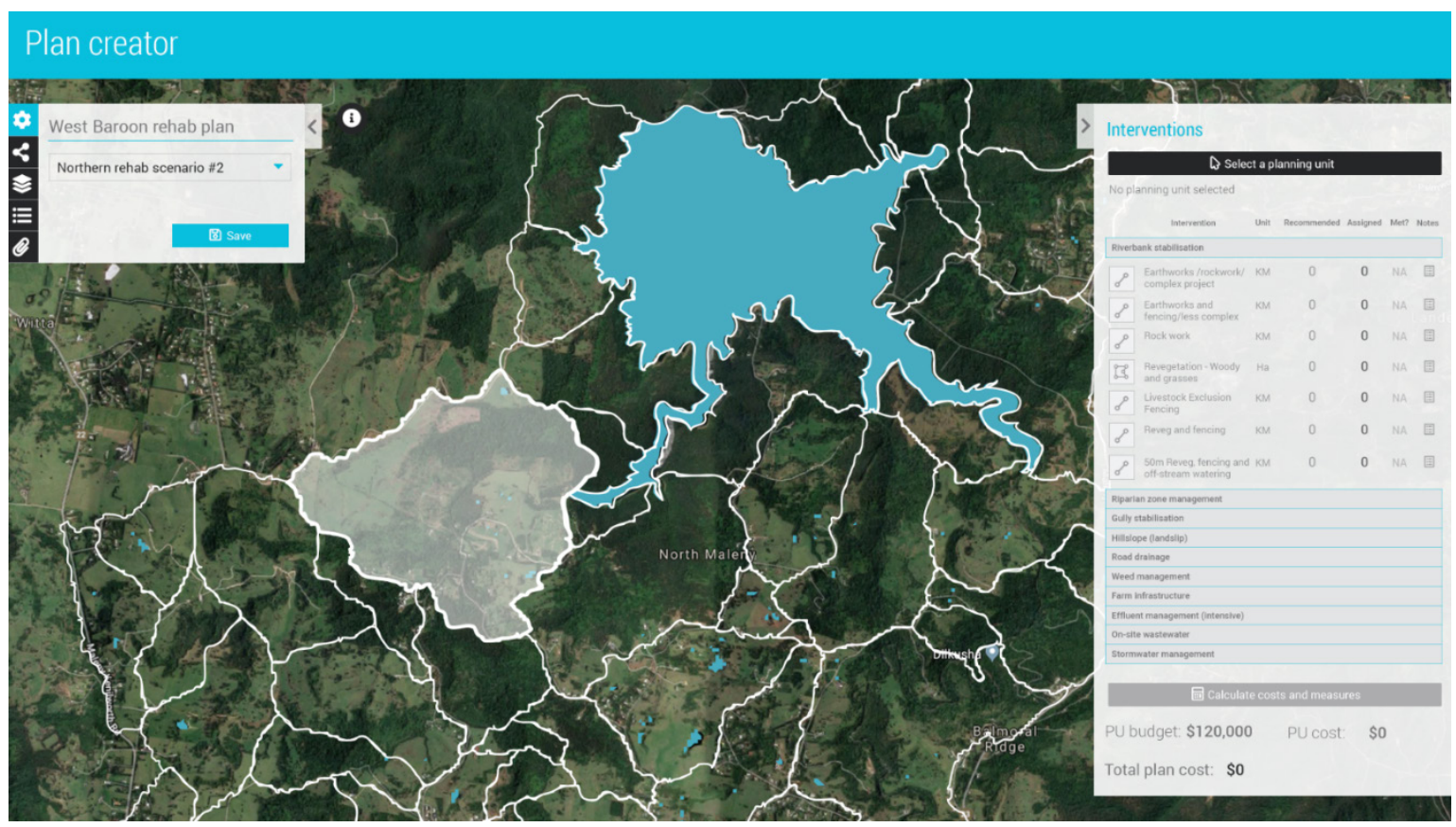

Figure 7. Operational view - annotated map to define where work can be conducted.

\section{UPDATE MODEL INPUTS}

At the completion of the NRM investment planning process some on-ground works are implemented. The next time that the NRM investment planning occurs (often annually), it is important to capture where work has been carried out in the intervening period. It is also important to be able to track the ongoing performance of an intervention as well as update the base case with improvements in knowledge/data, such as more accurate estimations of the attenuation of microbial pathogens or that a certain water treatment plant upgrade has resulted in improved treatment capability. The 'as-constructed' may not directly reflect the workplan as opportunities change through the implementation period. The CIDSS approach to this problem is to allow the annotated maps to be updated with an 'as-constructed' status representing what work was actually completed. The as-constructed layer can then be committed back to the source data to represent an updated catchment risk level to drinking water safety.

In practice the representation of the 'as-constructed' on-ground investment is via a natural assets management system which is maintained to ensure that on-ground works are monitored and maintained to ensure they continue to function as intended to reduce catchment risks.

\section{CONCLUSIONS}

The CIDSS closes the loop in the modelling-planning-operationalise workflow sequence by using a range of interfaces to interact with (i) collated data and parameterized model environment, (ii) scenario development and execution, (iii) solution interrogation and pragmatic refinement of the optimized scenario result. Interfaces allow communication of selected scenario solutions between policy makers and stakeholders which creates improved understanding and allows a broader engagement with a quantitative modelling approach to NRM investment planning.

\section{ACKNOWLEDGEMENTS}

We are grateful for the review and support provide by Dr Anthony Ladson and David Waters. There comments have greatly improved the CIDSS functionality. Many thanks to Thomas Lynn and Ryan Conte who have made the CIDSS a reality through their software development skills. 
Marsh et al., Closing the loop on modelling's role in natural resource investment planning

\section{REFERENCES}

ABS (2007) Natural resource management on Australian farms. Australian Bureau of Statistics, Canberra, Australia, publication No 4620.0

Herron, N.F., Barrett, T.W., Cuddy, S.M. (2008) Linking Catchment Environmental Planning to On-Ground Investment: the SCaRPA DSS. International Congress on Environmental Modelling and Software. 178. htps://scholarsarchive.byu.edu/iemssconference/2008/all/178

RMCG (2017) Improving data alignment, Department of the Environment and Energy, Canberra, Australia

QAO (2015) Managing water quality in Great Barrier Reef catchments Report 20: 2014-15, Queensland Audit Office, Brisbane, Australia

Schwartz, M.W, Cook, C.N., Pressey, R.L, Pullin, A.S., Runge, M.C., Salafsky, N., Sutherland, W.J., Williamson, M.A. (2019) Decision support frameworks and tools for conservation. Conservation Letters 11(2) 1-12

Summerell, G.K., Barrett, T., Grieve, A.M., Dunn, M., Moerkerken, L., Love, J. (2011) Implementing decision support for natural resource management agencies - the SCaRPA experience. $19^{\text {th }}$ International Congress on Modelling and Simulation, Perth, Australia, 12-16 December. http://mssanz.org.au/modsim2011 